\title{
Endoprosthetic treatment of primary bone sarcomas with pathological fractures
}

\author{
Dr Thomas L Hilton MBChB(UCT), DA(SA), DipPEC(SA), FCS(Orth), MMed(UCT) \\ Dr Keith Hosking MBChB(UCT), FCOrth(SA) \\ Vincent Pallotti Life Orthopaedic Hospital, and Princess Alice Orthopaedic Unit, \\ Groote Schuur Hospital, University of Cape Town
}

\author{
Correspondence: \\ Dr TL Hilton \\ 11 Clive Street \\ Vredehoek \\ 8000 Cape Town, South Africa \\ Email: tlhilton@hotmail.com \\ Cell: 0027 (82) 7967608
}

\begin{abstract}
Background: Primary bone sarcomas that are associated with a pathological fracture are rare and as a group have a worse prognosis than their non-fractured counterparts.

Questions/purposes: Traditionally limb ablation was advised; however, recent evidence suggests that limb salvage is a safe and acceptable form of treatment for both surgeon and patient.

Patients and methods: We present a retrospective review of a series of six patients referred to our unit with pathological fractures. These were treated by initial traction and neo-adjuvant chemotherapy where indicated with subsequent resection and endoprosthetic replacement.

Results: The age range of our series is from 20 to 81 years, with four males and two females. All had distal femur involvement with a $60 \%$ incidence of osteosarcoma and $40 \%$ chondrosarcoma. Three patients required total femur resection due to extensive tumour involvement. Our results show $100 \%$ of patients had clear margins at postoperative histology. Due to the aggressive nature of these types of tumours they carry a worse long-term prognosis and as such we had three deaths in our series. One patient died of a myocardial infarction post-operatively, and two patients developed lung metastases and died 2 years later.

Conclusion: Our conclusion is that with careful planning, a safe margin can be achieved. Endoprosthetic replacement allows for rapid reconstruction and mobilisation in this group of patients facilitating further oncological management.
\end{abstract}

Key words: pathological fracture, sarcoma, limb salvage, endoprosthetic replacement

http://dx.doi.org/10.17159/2309-8309/2016/v15n2a7

\section{Introduction}

Primary bone sarcomas that are associated with a pathological fracture (Figures 1-4) are rare and as a group have a worse prognosis than their non-fractured counterparts. ${ }^{1-5}$ It was previously thought that this problem should be treated with amputation to prevent local recurrence, reduce incidence of metastases and improve survival., ${ }^{4,6-8}$ More recent studies have shown that careful selection of patients, initial immobilisation, and neo-adjuvant chemotherapy where indicated, leads to fracture healing and facilitates adequate resection of margins subsequently. ${ }^{1,9-16}$
This treatment results in comparable survival rates to amputation with the advantages of a cosmetically and functionally superior limb. ${ }^{10}$

\section{Materials and methods}

We performed a retrospective review of a series of six patients referred to our unit with pathological fractures from 2009 to 2014. The patients were admitted and treated with bed rest and skin traction until fracture union. Skeletal traction was avoided to minimise septic complications. 


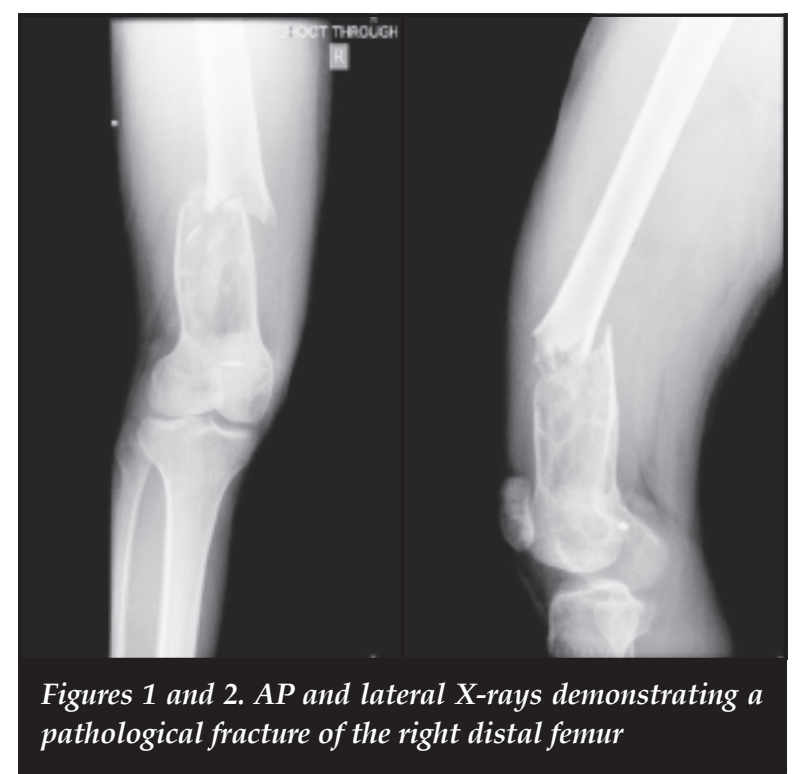

During their admission, an MRI scan of the entire involved femur was done to assess the site and size of the lesion, its proximity to neurovascular structures, and to exclude skip and satellite lesions. A CT chest and abdomen including a nuclear medicine bone scan was performed to stage the patient and assess for the presence of metastases. A needle biopsy for histological confirmation of diagnosis and grading of the tumour was performed.

Osteosarcoma patients underwent neo-adjuvant chemotherapy prior to surgery to facilitate fracture union and tumour shrinkage. No patient received radiotherapy prior to surgery.

Patients were consented and counselled about the possibility of an amputation if the macroscopic appearance of the tumour was unfavourable at the time of surgery.

Surgical treatment included limb salvage surgery with wide excision of the tumour and endoprosthetic replacement (Figures 5-7). Rehabilitation was per the unit's protocol.

\section{Results}

Of the six patients, the age range was from 20 to 81 years, with four males and two females. The follow-up period was at a median of 12 months from time of presentation (range min 5, max 61). All patients had distal femur involvement with a $60 \%$ incidence of osteosarcoma and a $40 \%$ incidence of chondrosarcoma. Four of the six patients were diagnosed with a primary bone sarcoma and pathological fracture at their index presentation. Two of the patients were undergoing neoadjuvant chemotherapy for their primary bone sarcoma at the time of fracture. No patients sustained an intra-operative fracture. Three patients required total femur resection due to extensive involvement of their disease.

MRI was essential in surgical planning to ascertain the degree of soft tissue involvement, intramedullary extent, as well as proximity to the neurovascular bundle.

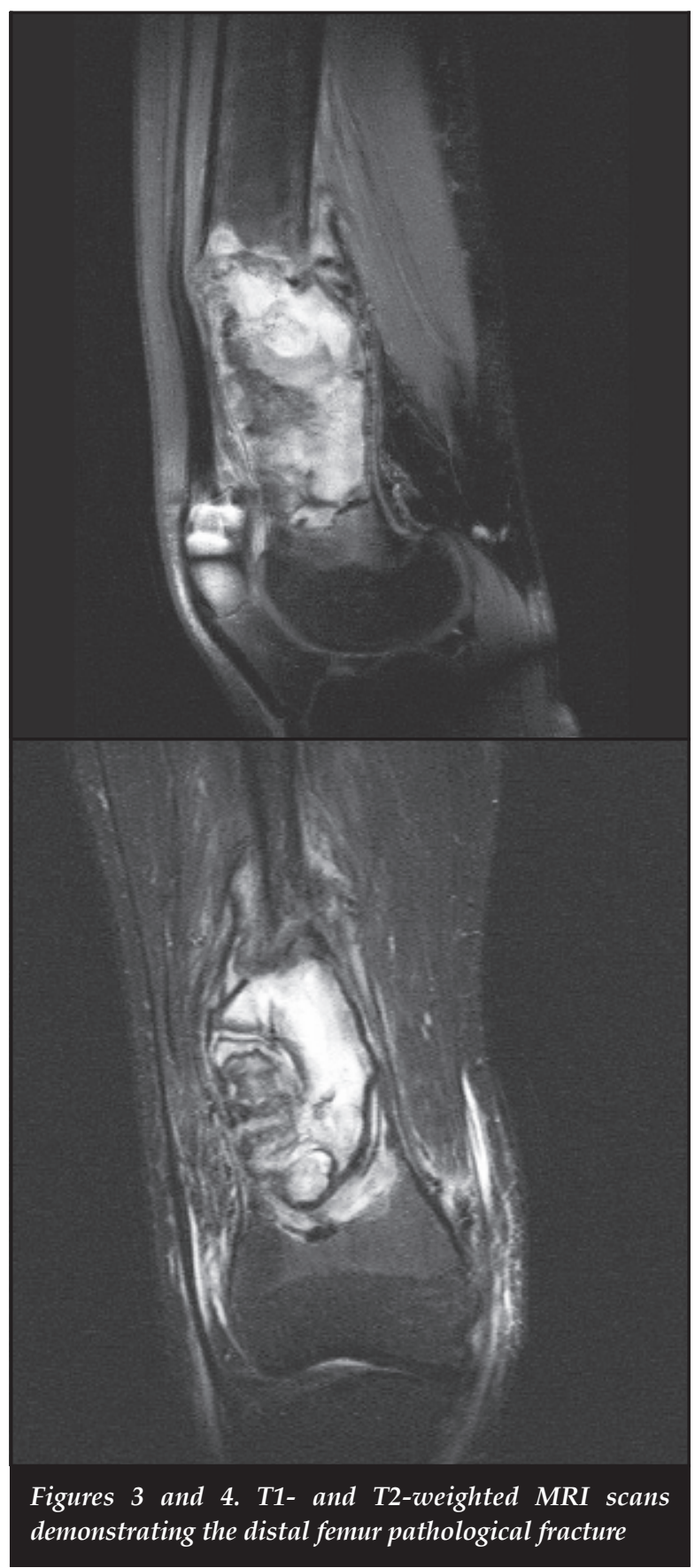

All of the sarcomas were high-grade and extra-compartmental in nature. A marginal or wide excision margin was achieved in all cases. Due to the aggressive nature of these high-grade sarcomas, they carry a worse long-term prognosis and we had three deaths in our series. One patient died of a myocardial infarction post-operatively, and two patients developed lung metastases, undiagnosed at initial presentation and died 2 years after completion of initial treatment. One of the two patients who died from the disease developed local recurrence 2 years after initial presentation and underwent limb ablation. 


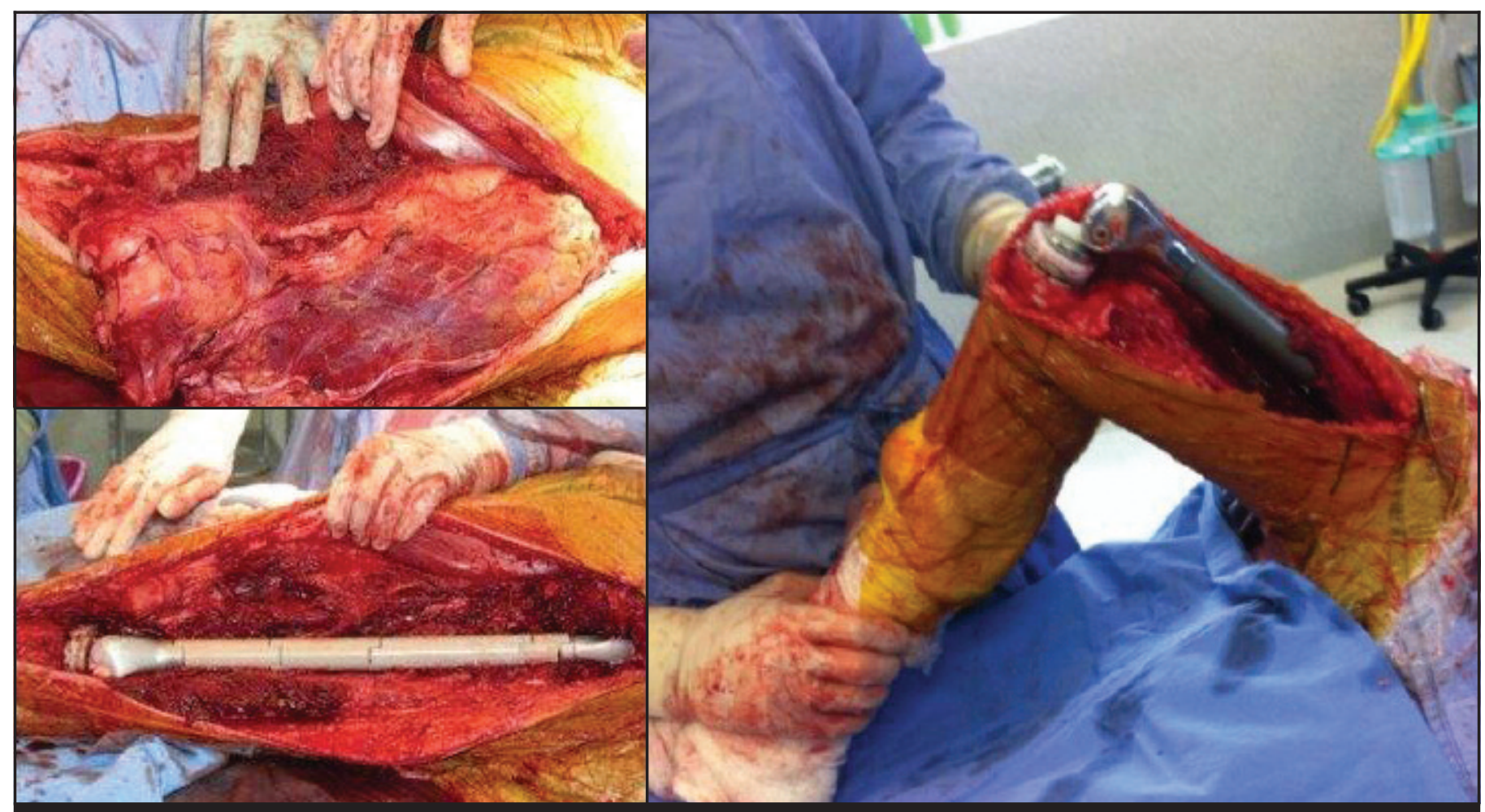

Figures 5, 6 and 7. Intra-operative image of excised tumour and endoprosthetic insertion

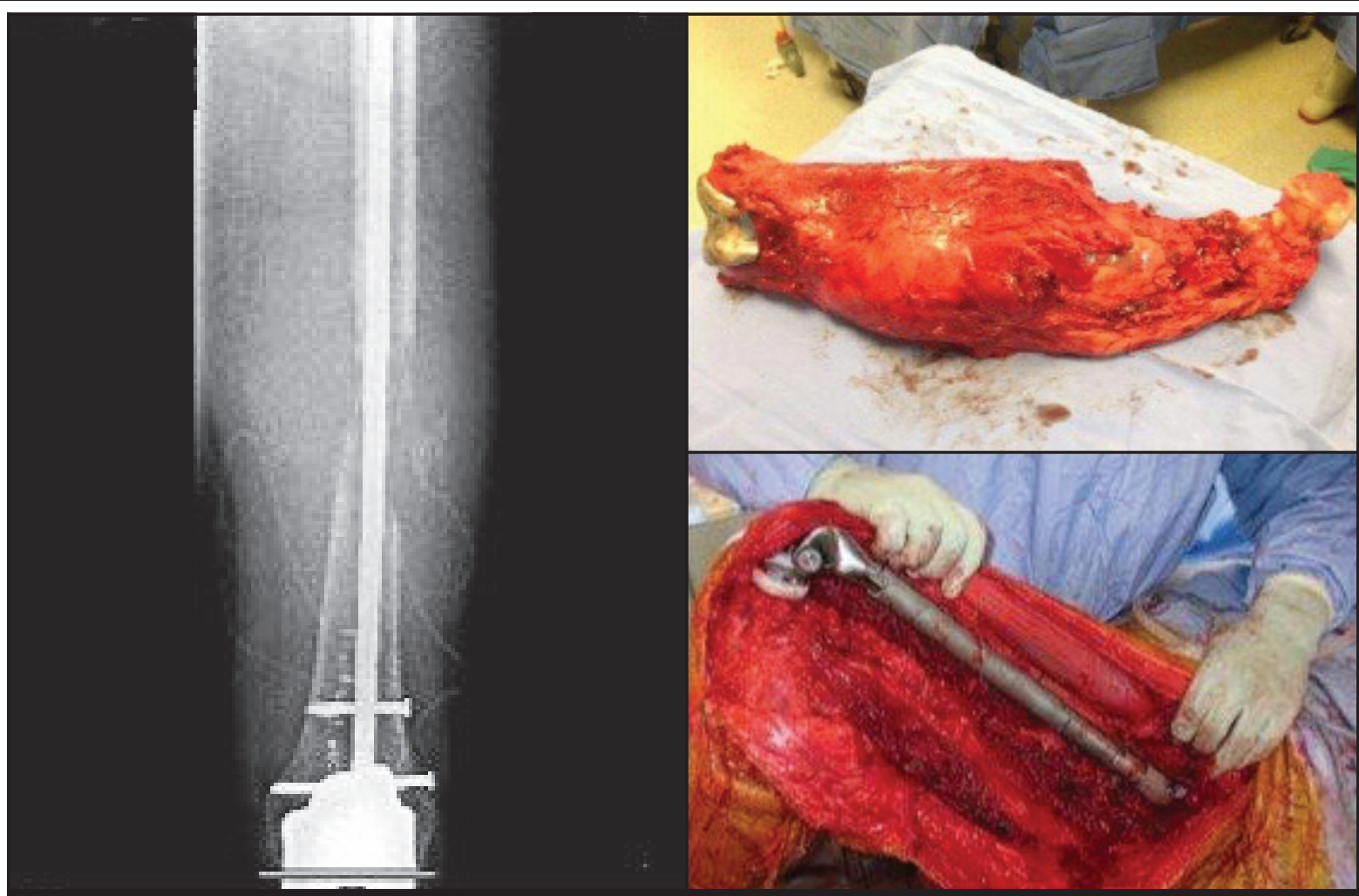

Figures 8, 9 and 10. X-ray and intra-operative images of a primary sarcoma with pathological fracture treated with intramedullary nailing. The result was extensive spread of tumour throughout the femur, necessitating endoprosthetic replacement. 


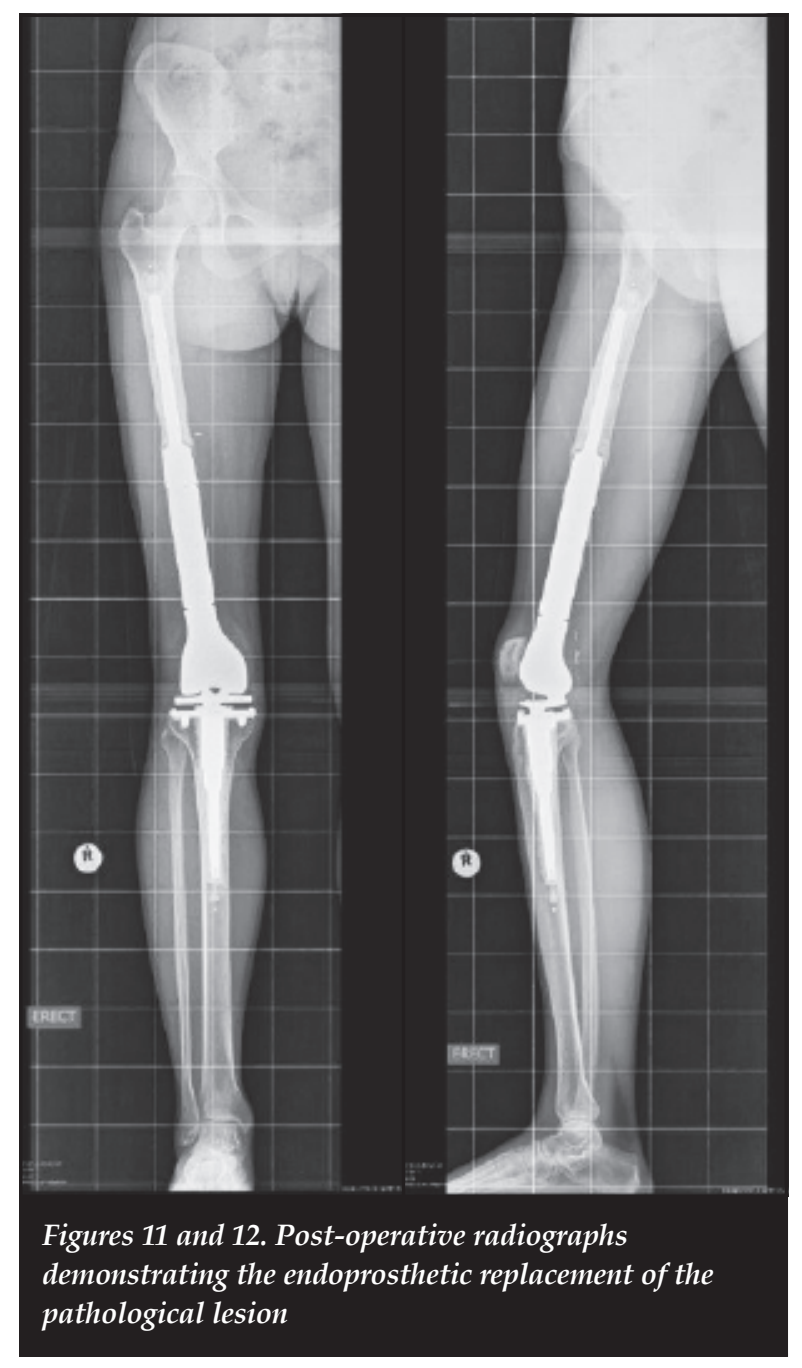

\section{Discussion}

The principal goal in the management of patients who have a primary bone sarcoma is prolonging their survival. ${ }^{13}$ Most studies comparing amputation and limb salvage report no adverse effects of the long-term survival of patients with primary bone sarcomas treated with limbsparing surgery. The current trend for the treatment of pathological fractures as a consequence of primary bone sarcomas is still towards limb salvage; however, this has not always been the case. Amputation was the most common procedure of choice. It was recommended because it was thought that the local fracture haematoma disseminated tumour cells into the adjacent tissue and joints. Also, it was thought that damage to the microvascular circulation facilitated metastases. Factors that changed the trend towards limb salvage included the efficacy of neo-adjuvant chemotherapy, fracture union during pre-operative chemotherapy, and the newer functional reconstructive modalities of treatment and imaging. ${ }^{13,17}$

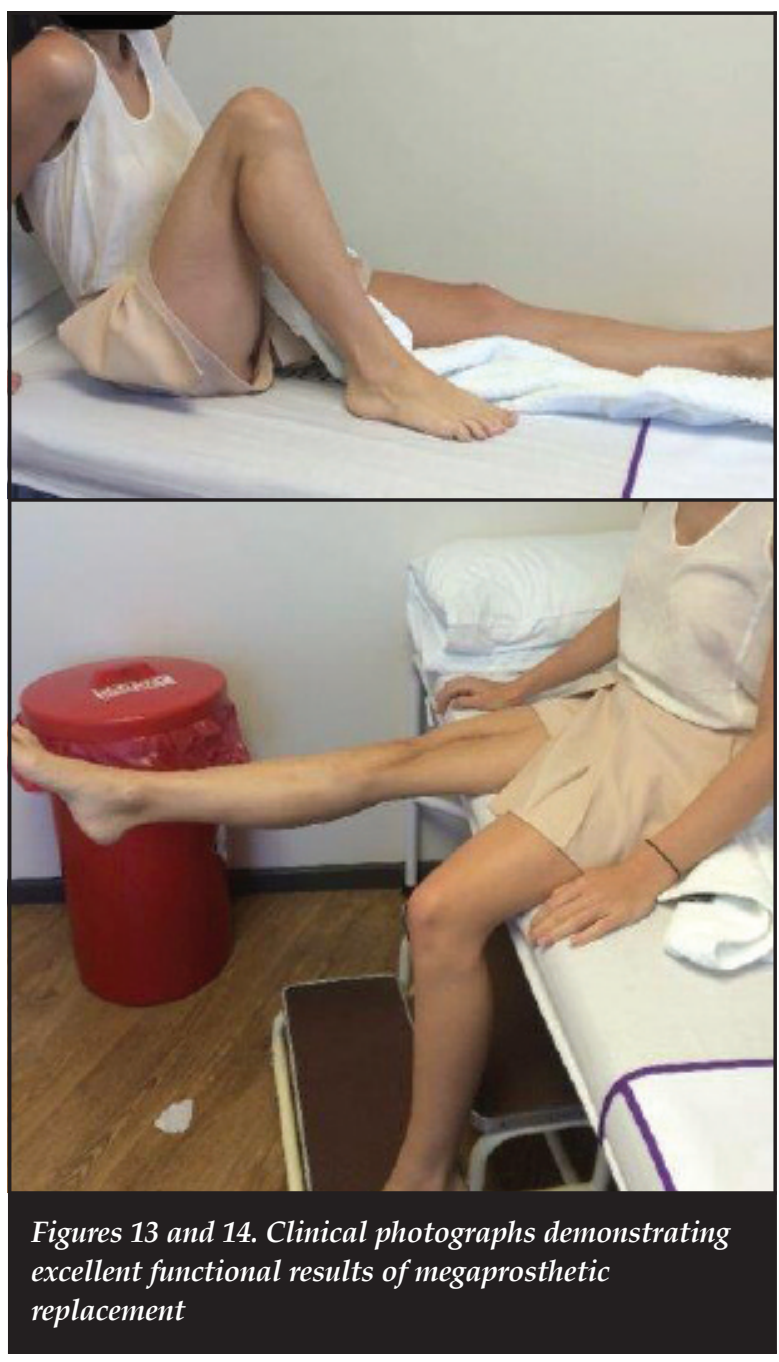

Pathological fractures occur due to the high-grade and lytic nature of these sarcomas. Fractures can occur spontaneously or after minimal trauma, because of the poor structural properties of the bone affected. The pathological bone often exhibits high cellularity, poor differentiation and significant loss of bone matrix..$^{18}$ Stress or mechanical weakness caused by diagnostic biopsy and necrosis of tumour after chemotherapy may contribute to development of fractures. ${ }^{19}$

The incidence of a pathological fracture either at diagnosis or during the peri-operative period is between $5 \%$ and $10 \% \cdot{ }^{17,20}$ It is important to both make a prompt diagnosis of a pathological fracture and not to miss the diagnosis in the first place, as internal fixation of these fractures may result in tumour spread and impairment of definitive treatment (Figures 8-12). ${ }^{9}$

As mentioned earlier, amputation was the procedure of choice for these injuries to ensure optimal outcome in terms of recurrence and survival. This is supported by studies that show that amputation produces a better 
outcome in the eradication of the local tumour than limb salvage. ${ }^{9,21,22}$ However, recent studies have shown no difference in the outcome after limb salvage, including tumour recurrence, the development of metastases and overall survival in patients who presented with a fracture and those who developed a fracture with treatment. ${ }^{23}$

Neo-adjuvant chemotherapy improves overall survival in osteosarcoma assisting with tumour shrinkage and union. ${ }^{1724}$ Without chemotherapy survival rates are as low as $20 \%, 25,26$ but with chemotherapy increase to $60 \%$ overall. $^{27-29}$ Patients are treated with bed rest and skin traction while undergoing chemotherapy until fracture union. This aids in the manipulation of the limb during the definitive surgery. ${ }^{13}$ Radiation post limb salvage is not recommended as it has not been shown to be successful in preventing local recurrence or metastases, and it may compromise success of definitive surgery and any potential further surgery. ${ }^{30}$

MRI is extremely useful in the planning of the definitive surgery. A thorough MRI assessment before and after chemotherapy is useful to determine the extent of the initial haematoma and the presence of skip or satellite lesions. This helps to plan the extent of the tumour excision and type of endoprosthetic required. Limb salvage is usually offered unless the tumour cannot be separated from important neurovascular structures, has grossly invaded a joint or where resection of too much muscle would result in a functionless limb. ${ }^{9}$ The reconstructive option is usually with a megaprosthetic or allograft prosthetic composite, which gives the patient a good cosmetic and functional result (Figures 13 and 14). In keeping with Phemister's law that bone sarcomas have a predilection to occur around joints due to the relative increase in blood supply, the majority of these primary bone sarcomas that sustain pathological fracture occur around the knee and usually involve the joint, necessitating its inclusion in the prosthesis. Sarcomas tend to spread along muscle compartments and are limited by fascial planes. The pathological fracture is usually a low energy process with less soft tissue contamination than conventional fractures, and surgery within certain tissue planes is possible.

The incidence of local recurrence has been reported to be approximately $19 \%$, which is consistent with our study, which showed a recurrence rate of $17 \% .^{9}$ The number of deaths unrelated to the tumour or its treatment was slightly higher in our series $(17 \%)$ compared to reported literature of approximately $8 \%$; however, this may be due to our small numbers. ${ }^{9}$ Deaths related to the tumour in our series were $33 \%$, compared to $47 \%$ reported in the literature. ${ }^{9}$ The prognosis for these patients is guarded from the outset due to the often aggressive and high-grade nature of the sarcomas that are associated with pathological fracture. In the past the prognosis of patients presenting with this diagnosis was uncertain; however, recent studies suggest that with modern treatment it is similar to those without fracture.., 31

\section{Conclusion}

Our conclusion is that one can achieve safe local eradication of the tumour and perform limb salvage surgery for primary bone sarcomas with pathological fractures, without affecting patient morbidity or mortality while sparing the patient an amputation. The prognosis for these patients is poor from the outset, but the advantages of limb salvage for the patient are those of improved cosmetic and function gains.

\section{Compliance with Ethics Guidelines}

$\mathrm{T}$ Hilton and $\mathrm{K}$ Hosking declare that they have no commercial associations that might pose a conflict of interest in connection with the submitted article. Work for the article was performed at Groote Schuur Hospital and Vincent Pallotti Life Orthopaedic Hospital.

\section{References}

1. Bramer JA et al. Do pathological fractures influence survival and local recurrence rate in bony sarcomas? Eur J Cancer, 2007;43(13):1944-51.

2. Lee RK et al. Pathological fracture as the presenting feature in pediatric osteosarcoma. Pediatr Blood Cancer, 2013;60(7):1118-21.

3. Moradi B et al. The impact of pathological fractures on therapy outcome in patients with primary malignant bone sarcomas. Int Orthop, 2010;34(7):1017-23.

4. Puri A et al. Chondrosarcoma of bone: does the size of the tumor, the presence of a pathologic fracture, or prior intervention have an impact on local control and survival? J Cancer Res Ther, 2009;5(1):14-19.

5. Zeifang F, Sabo D, Ewerbeck V. [Pathological fracture in primary malignant bone tumors]. Chirurg, 2000;71(9):1121-5.

6. Godley K, Watts AC, Robb JE. Pathological femoral fracture caused by primary bone tumour: a populationbased study. Scott Med J, 2011;56(1):5-9.

7. Papagelopoulos PJ et al. Pathological fractures in primary bone sarcomas. Injury, 2008;39(4):395-403.

8. Scully SP et al. The surgical treatment of patients with osteosarcoma who sustain a pathologic fracture. Clin Orthop Relat Res, 1996;324:227-32.

9. Abudu A et al. The surgical treatment and outcome of pathological fractures in localised osteosarcoma. J Bone Joint Surg Br, 1996;78(5):694-98.

10. Bacci G et al. Nonmetastatic osteosarcoma of the extremity with pathologic fracture at presentation: local and systemic control by amputation or limb salvage after preoperative chemotherapy. Acta Orthop Scand, 2003;74(4):449-54.

11. Cui $\mathrm{Q}$ et al. Two case-reports of the limb salvage treatment of osteosarcoma consolidated with obvious pathological fractures. Pathol Oncol Res, 2011;17(4):973-79.

12. De Mattos CB, Binitie O, Dormans JP. Pathological fractures in children. Bone Joint Res, 2012;1(10):272-80.

13. Ebeid W, Amin S, Abdelmegid A. Limb salvage management of pathologic fractures of primary malignant bone tumors. Cancer Control, 2005;12(1):57-61. 
14. Li D, Cui Q, Wang L. [The effect of limb salvage on treating osteosarcoma with pathological fracture in two cases]. Zhongguo Хiu Fu Chong Jian Wai Ke Za Zhi, 2006;20(1):30-32.

15. Niu XH, Ding Y. [The surgical treatment and outcome of nonmetastatic osteosarcoma of the extremity with pathologic fractures]. Zhonghua Wai Ke Za Zhi, 2008;46(22):173033.

16. Xie L et al. Pathologic fracture does not influence local recurrence and survival in high-grade extremity osteosarcoma with adequate surgical margins. J Surg Oncol, 2012;106(7):820-25.

17. Jaffe $\mathrm{N}$ et al. Pathologic fracture in osteosarcoma. Impact of chemotherapy on primary tumor and survival. Cancer, 1987;59(4):701-709.

18. Present D, Bertoni F, Enneking WF. Osteosarcoma of the mandible arising in fibrous dysplasia. A case report. Clin Orthop Relat Res, 1986;204:238-44.

19. Clark CR et al. The effect of biopsy-hole shape and size on bone strength. J Bone Joint Surg Am, 1977;59(2):213-17.

20. Mulder J, Schutte HE, Kroon HM, Taconis WK. Intraosseous osteosarcoma: conventional type. Radiological Atlas of Bone Sarcomas, 1993;51-55.

21. Simon MA et al. Limb-salvage treatment versus amputation for osteosarcoma of the distal end of the femur. J Bone Joint Surg Am, 1986;68(9):1331-37.

22. Shin KH, Rougraff BT, Simon MA. Oncologic outcomes of primary bone sarcomas of the pelvis. Clin Orthop Relat Res, 1994;304:207-17.

23. Chandrasekar CR et al. Outcome of pathologic fractures of the proximal femur in nonosteogenic primary bone sarcoma. Eur J Surg Oncol, 2011;37(6):532-36.
24. Dubousset J, Missenard G, Kalifa C. Management of osteogenic sarcoma in children and adolescents. Clin Orthop Relat Res, 1991;270:52-59.

25. McKenna R, Schwinn CP, Soong KY et al. Sarcomata of osteogenic series (osteosarcoma, fibrosarcoma, chondrosarcoma, parosteal osteogenic sarcoma, and sarcomata arising in abnormal bone): an analysis of 552 cases. J Bone Joint Surg Am, 1966;48-A:1-26.

26. Elwood PC et al. Cardiovascular surveys in areas with different water supplies. Br Med J, 1971;2(5758):362-63.

27. Krischer JP et al. Nitrogen mustard, vincristine, procarbazine, and prednisone as adjuvant chemotherapy in the treatment of medulloblastoma. A Pediatric Oncology Group study. J Neurosurg, 1991;74(6):905-909.

28. Bramwell VH et al. A comparison of two short intensive adjuvant chemotherapy regimens in operable osteosarcoma of limbs in children and young adults: the first study of the European Osteosarcoma Intergroup. J Clin Oncol, 1992;10(10):1579-91.

29. Winkler $\mathrm{K}$ et al. Treatment of osteosarcoma: experience of the Cooperative Osteosarcoma Study Group (COSS). Cancer Treat Res, 1993;62:269-77.

30. Huvos AG et al. Telangiectatic osteogenic sarcoma: a clinicopathologic study of 124 patients. Cancer, 1982;49(8):1679-89.

31. O'Hara JM et al. An analysis of thirty patients surviving longer than ten years after treatment for osteogenic sarcoma. J Bone Joint Surg Am, 1968;50(2):335-54.

This article is also available online on the SAOA website (www.saoa.org.za) and the SciELO website (www.scielo.org.za). Follow the directions on the Contents page of this journal to access it. 